\title{
Heavy Higgs boson resonances and their decay into top quarks at the LHC
}

\author{
Werner Bernreuther ${ }^{a}$, Peter Galler ${ }^{* b}$, Clemens Mellein ${ }^{a}$, Zong-Guo Si ${ }^{c}$ and Peter Uwer ${ }^{b}$ \\ ${ }^{a}$ Institut für Theoretische Teilchenphysik und Kosmologie, \\ RWTH Aachen University, 52056 Aachen, Germany \\ ${ }^{b}$ Institut für Physik, Humboldt-Universität zu Berlin, 12489 Berlin, Germany \\ ${ }^{c}$ School of Physics, Shandong University, Jinan, Shandong 250100, China \\ E-mail: breuther@physik.rwth-aachen.de, galler@physik.hu-berlin.de, \\ mellein@physik.rwth-aachen.de, zgsi@sdu.edu.cn, uwer@physik.hu-berlin.de
}

\begin{abstract}
We investigate, within the type-II two-Higgs-doublet extension of the standard model (SM), the impact of heavy neutral Higgs boson resonances with unsuppressed Yukawa couplings to top quarks on top-quark pair production at the LHC at next-to-leading order (NLO) in the strong coupling constant. We take into account the resonant Higgs boson contributions, the non-resonant SM $t \bar{t}$ continuum and the interference of these two contributions. The NLO QCD corrections to heavy Higgs production and the interference contributions are calculated in the large top-quark mass $\left(m_{t}\right)$ limit, including an effective K-factor rescaling. Our evaluation of the QCD-Higgs interference is focused on the Higgs resonance region. Using representative $C P$-conserving as well as $C P$-violating parameter scenarios phenomenological results are presented for different observables.
\end{abstract}

Fourth Annual Large Hadron Collider Physics

13-18 June 2016

Lund, Sweden

${ }^{*}$ Speaker. 


\section{Introduction}

After the $125 \mathrm{GeV}$ spin-zero resonance has been experimentally established as a Higgs boson with Standard-Model like couplings to fermions and vector bosons [1, 2, 3, 4], a central research issue at the LHC is the question whether additional spin-zero bosons exist. We are interested in heavy Higgs bosons with masses $m_{\phi}>2 m_{t}$ and unsuppressed Yukawa couplings to the top quark that can appear as resonances in the $t \bar{t}$ decay channel. Experimental searches for such resonances have been performed by ATLAS [5] and CMS [6, 7] but so far no signals above the SM continuum were detected. However, due to experimental (and theoretical) uncertainties these measurements are limited in constraining heavy Higgs models. Furthermore, an important effect in the decay of heavy Higgs bosons to $t \bar{t}$ is the interference with the $t \bar{t}$ continuum that can have a strong influence on the detectability of these resonances. On the theoretical side the production of heavy Higgs bosons and decay into top-quark pairs has been studied until recently only at leading order (LO) in QCD. NLO corrections as calculated very recently in $[8,9]$ have not been taken into account in the experimental analysis. In this contribution we report on the calculation [8] of the NLO QCD corrections to this process, presenting results for inclusive and differential cross sections at a center of mass energy of $\sqrt{s}=13 \mathrm{TeV}$ at the LHC. While at LO the full $m_{t}$ dependence is kept, the NLO corrections are calculated in the heavy top-quark limit which induces an effective coupling between the Higgs bosons and gluons. Because relevant contributions from the heavy Higgs bosons to $t \bar{t}$ production are only expected in the resonant region, we restricted our computation of the NLO QCD corrections to this region which simplifies the calculation further. Our analysis is not restricted to a particular model and can be applied to a wide spectrum of new physics models involving heavy spin- 0 bosons that couple to $t \bar{t}$. However, the choice of a specific model is in order since the decay widths of the Higgs bosons should be taken into account in a consistent fashion. This is not only important for preserving unitarity but also because the effects of heavy Higgs bosons on the $t \bar{t}$ production sensibly depend on the bosons' decay widths. Hence, we have chosen a UV-complete theory of an extended Higgs sector, the type-II two-Higgs-doublet model (2HDM), and the decay widths are determined by the independent parameters of the model.

This article is organized as follows. In Sec. 2 we give a brief overview on aspects of the type-II $2 \mathrm{HDM}$ relevant for the following sections. We study three $2 \mathrm{HDM}$ parameter scenarios that are introduced in Sec. 3. Leading order results are shown in Sec. 4 in order to exhibit the main features of heavy Higgs resonances in $t \bar{t}$ production. In Sec. 5 our NLO calculation [8] is outlined. In Sec. 6 we show phenomenological results at NLO QCD for different observables and for the three scenarios introduced in Sec. 3. We conclude in Sec. 7.

\section{Type-II two-Higgs-doublet model}

As mentioned in the introduction we choose the type-II two-Higgs-doublet model as a Higgs sector extension of the SM. It allows us to consistently incorporate the already discovered SM Higgs boson with $m_{h}=125 \mathrm{GeV}$ as well as additional heavy spin-0 bosons which can show up as resonances in the $t \bar{t}$ decay channel. Because of strong experimental constraints on flavor changing neutral currents we choose to study a flavor conserving 2HDM. In particular, we focus on the type-II $2 \mathrm{HDM}$ in which tree-level neutral flavor conservation is realized by coupling the right- 
chiral down-type quarks as well as the charged leptons only to the SU(2)-Higgs doublet $\Phi_{1}$ and the right-chiral up-type quarks to the $\mathrm{SU}(2)$-Higgs doublet $\Phi_{2}$. For a detailed description of the $2 \mathrm{HDM}$ we refer to the literature, e.g. [10].

The two Higgs doublets are given in the unitary gauge and after electroweak symmetry breaking by:

$$
\Phi_{1}=\left(-H^{+} \sin \beta, \frac{1}{\sqrt{2}}\left(v_{1}+\varphi_{1}-i A \sin \beta\right)\right)^{T}, \quad \Phi_{2}=\left(H^{+} \cos \beta, \frac{1}{\sqrt{2}}\left(v_{2}+\varphi_{2}+i A \cos \beta\right)\right)^{T},
$$

where $v_{1}$ and $v_{2}$ are the vacuum expectation values of the two Higgs doublets with $v=\sqrt{v_{1}^{2}+v_{2}^{2}}=$ $246 \mathrm{GeV}$ and $\tan \beta=v_{2} / v_{1}$. The $C P$-even Higgs bosons are denoted by $\varphi_{1}$ and $\varphi_{2}$ and the $C P$-odd Higgs boson is denoted by $A$. The neutral $C P$-even Higgs bosons can mix with each other and their mass eigenstates are combinations of $\varphi_{1}$ and $\varphi_{2}$ parametrized by the mixing angle $\alpha$. Furthermore, there is a physical charged Higgs boson denoted by $H^{ \pm}$which however plays a minor role in our analysis. The $2 \mathrm{HDM}$ also allows for $C P$ violation in the Higgs sector. In this case the three neutral Higgs bosons can mix with each other such that the mass eigenstates $\phi_{i}(i=1,2,3)$ are not $C P$ eigenstates. The mixing is described by an orthogonal mixing matrix $R$ that can be parametrized by three mixing angles $\alpha_{i}(i=1,2,3)$ :

$$
\left(\begin{array}{c}
\phi_{1} \\
\phi_{2} \\
\phi_{3}
\end{array}\right)=R\left(\alpha_{1}, \alpha_{2}, \alpha_{3}\right)\left(\begin{array}{c}
\varphi_{1} \\
\varphi_{2} \\
A
\end{array}\right)
$$

For the $C P$-violating $2 \mathrm{HDM}$ we choose

$$
m_{1}, m_{2}, m_{3}, m_{+}, \alpha_{1}, \alpha_{2}, \alpha_{3}, \tan \beta, v
$$

as input parameters. Here $m_{i}(i=1,2,3)$ denote the masses of the neutral Higgs bosons $\phi_{i}$ and $m_{+}$ the mass of $H^{+}$. For the $C P$ conserving $2 \mathrm{HDM}$ the number of independent parameters of the Higgs potential is reduced and we choose the following set:

$$
m_{1}, m_{2}, m_{3}, m_{+}, \alpha, \tan \beta, v
$$

where $m_{1}$ and $m_{2}$ are the masses of the scalars with $m_{1}<m_{2}$ and $m_{3}$ is the mass of the pseudoscalar.

\section{Scenarios}

We investigate three parameter scenarios within the $2 \mathrm{HDM}$, two of them correspond to $C P$ conservation and one to $C P$ violation by the neutral Higgs-boson interactions. The parameters are chosen as follows:

a) The mass of the lightest neutral Higgs boson $\phi_{1}$ of the model is put to $m_{1}=125 \mathrm{GeV}$ and its couplings are chosen to be equal or close to the SM Higgs couplings. Equality (i.e., the so-called alignment limit) can be achieved only in the $C P$-conserving scenarios. In our $C P$-violating scenario the three neutral Higgs-boson mass eigenstates are $C P$-mixed states. 
Thus the couplings of $\phi_{1}$ differ from the SM Higgs couplings. Yet it is possible to choose the parameters such that the deviations of the couplings of $\phi_{1}$ from the respective SM Higgs couplings are within the experimentally allowed range.

b) The Yukawa couplings to top quarks are enhanced. In order to estimate the possible size of the effect in $t \bar{t}$ production we choose $\tan \beta=0.7$ which leads to top-Yukawa couplings of the neutral Higgs bosons that are still in accord with experimental constraints. This choice constrains also the mass of the charged Higgs boson to $m_{+}>720 \mathrm{GeV}[11,12,13]$.

c) The masses $m_{2}, m_{3}$ of the heavy Higgs bosons are chosen to be larger than twice the topquark mass, because we are interested in the resonant production of $\phi_{2}, \phi_{3}$ and their decay to top-quark pairs.

The choice of $\tan \beta=0.7$ leads to a suppression of the down-type Yukawa couplings. Thus in Higgs production by gluon fusion not only the contribution of the light quarks but also that of the $b$ quark can be safely neglected.

In the $C P$-conserving scenarios, the alignment limit is realized in our parametrization of $R$ by putting $\alpha_{1}=\beta$. Moreover, $\alpha_{2}=\alpha_{3}=0$. Thus all Yukawa couplings are determined by $\tan \beta$ only. In the alignment limit the coupling of $\phi_{1}$ to weak vector bosons is the same as in the SM. Therefore, the couplings of $\phi_{2}, \phi_{3}$ to $W W$ and $Z Z$ are zero. This implies that $\phi_{2}, \phi_{3} \rightarrow t \bar{t}$ is the dominant decay mode of the heavy Higgs bosons.

The masses of the Higgs bosons are free parameters of the 2HDM. The heavy Higgs boson masses are chosen as follows: In the $C P$-conserving case we distinguish two scenarios, one with almost degenerate masses (scenario 1) and the other one with non-degenerate masses (scenario 2). In the $C P$-violating scenario (scenario 3) the masses of the heavy Higgs bosons are also chosen to be non-degenerate. The width of the Higgs bosons in the respective scenarios are fixed by the input parameters and have to be calculated at NLO (for details see [8] and references therein). Table 1 summarizes the input parameters including the corresponding decay widths and Yukawa couplings for all three scenarios.

\section{Leading order results}

Within the type-II 2HDM we study the resonant production of heavy neutral Higgs bosons and their decay into top-quark pairs,

$$
p p \rightarrow \phi_{2,3} \rightarrow t \bar{t} X
$$

including the SM background process

$$
p p \rightarrow t \bar{t} X
$$

as well as the interference of the amplitudes of these two processes. We investigate this reaction to leading order in the strong coupling constant $\alpha_{s}$ in order to illustrate the main features of heavy Higgs resonances in $t \bar{t}$ production before we turn to the NLO corrections in the next section. An observable that is sensitive to resonant heavy Higgs bosons in top-quark pair production is the $t \bar{t}$ invariant mass $M_{t \bar{t}}$. In Fig. 1 the $M_{t \bar{t}}$ distribution is shown for $t \bar{t}$ production by QCD (black) and for the sum of the QCD and Higgs-boson contributions (red) for scenario 3. In the lower plot different 


\begin{tabular}{|c|c|c|c|c|}
\hline & & scenario 1 & scenario 2 & scenario 3 \\
\hline \multirow{9}{*}{ 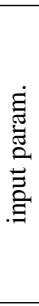 } & $\tan \beta$ & 0.7 & 0.7 & 0.7 \\
\hline & $v[\mathrm{GeV}]$ & 246 & 246 & 246 \\
\hline & $m_{+}[\mathrm{GeV}]$ & $>720$ & $>720$ & $>720$ \\
\hline & $m_{1}[\mathrm{GeV}]$ & 125 & 125 & 125 \\
\hline & $m_{2}[\mathrm{GeV}]$ & 550 & 550 & 500 \\
\hline & $m_{3}[\mathrm{GeV}]$ & 510 & 700 & 800 \\
\hline & $\alpha_{1}$ & $\beta$ & $\beta$ & $\beta$ \\
\hline & $\alpha_{2}$ & 0 & 0 & $\pi / 15$ \\
\hline & $\alpha_{3}$ & 0 & 0 & $\pi / 4$ \\
\hline \multirow{8}{*}{ 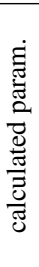 } & $\overline{\Gamma_{2}[\mathrm{GeV}]}$ & 34.56 & 34.49 & 36.55 \\
\hline & $\Gamma_{3}[\mathrm{GeV}]$ & 49.28 & 75.28 & 128.16 \\
\hline & $a_{t 1}$ & 1 & 1 & 0.978 \\
\hline & $b_{t 1}$ & 0 & 0 & 0.297 \\
\hline & $a_{t 2}$ & 1.429 & 1.429 & 0.863 \\
\hline & $b_{t 2}$ & 0 & 0 & 0.988 \\
\hline & $a_{t 3}$ & 0 & 0 & -1.157 \\
\hline & $b_{t 3}$ & 1.429 & 1.429 & 0.988 \\
\hline
\end{tabular}

Table 1: 2HDM parameter settings for scenarios 1-3. The SM-like Higgs boson $\phi_{1}$ has a decay width $\Gamma_{1} \approx 4 \mathrm{MeV}$. It plays no role in our analysis.

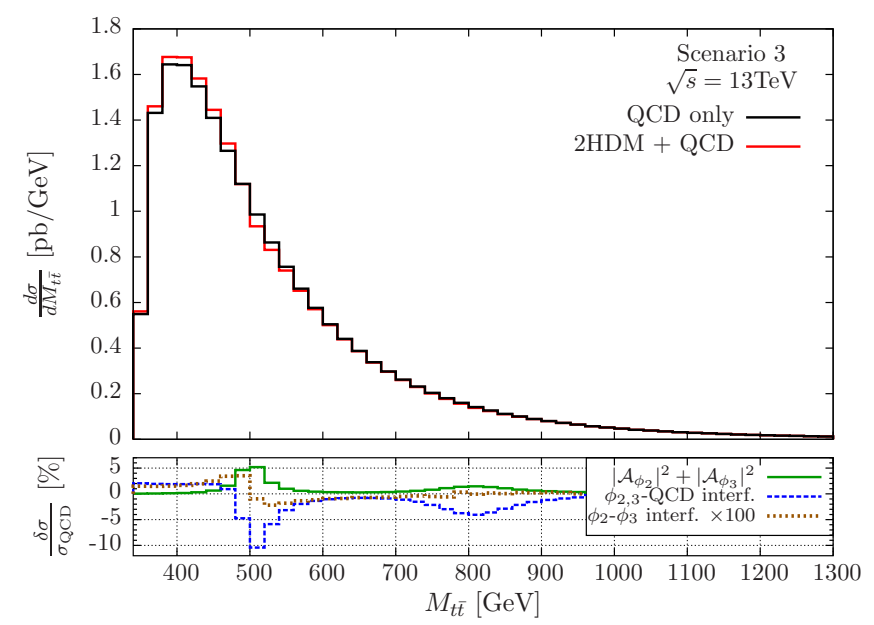

Figure 1: Upper plot: $M_{t \bar{t}}$ distribution at LO for the QCD background (black) and the sum of the QCD and Higgs contributions (red) for scenario 3. Lower plot: Ratio Higgs/QCD for different contributions to the full $M_{t \bar{t}}$ distribution. These are the sum of the squared amplitudes of the two heavy Higgs bosons (solid, green), the interference with the QCD background (dashed, blue) and the interference of the two heavy Higgs-boson amplitudes (dotted, brown).

pieces of the Higgs-boson contributions normalized to the QCD background are displayed. From this plot one can draw the following conclusions:

- The effect of the heavy Higss bosons in $t \bar{t}$ production is significant only in the resonant region. In Fig. 1 we show the contribution to the $M_{t \bar{t}}$ distribution of the two heavy Higgs bosons within our 2HDM parameter scenario 3 where $m_{2}=500 \mathrm{GeV}$ and $m_{3}=800 \mathrm{GeV}$. The resonant structures are clearly visible in these regions, especially in the lower plot, e.g. the green curve that displays the squared amplitudes of the $\phi_{2}$ and $\phi_{3}$ contributions. Away from the resonant region the effects of the heavy Higgs bosons are very small.

- The interference of the 2HDM amplitude with the QCD background amplitude is shown in 
blue in the lower plot of Fig. 1. The interference is mostly negative and its magnitude is of the same order as the squared Higgs amplitudes (green curve). This shows that the interference with the background has a strong effect on the shape of the $M_{t \bar{t}}$ distribution and hence can not be neglected. The sum of the squared Higgs amplitudes and interference term leads to a peak-dip structure around $500 \mathrm{GeV}$ and a dip around $800 \mathrm{GeV}$. This fact has important consequences for the search and detection of a heavy Higgs boson signal.

- In the $C P$-violating scenario 3 the Higgs bosons $\phi_{j}$ are $C P$ mixtures. As a consequence the amplitudes $g g \rightarrow \phi_{j} \rightarrow t \bar{t}$ interfere. This contribution to the $M_{t \bar{t}}$ distribution is shown as brown curve in Fig. 1 . Note that in this plot the $\phi_{2}-\phi_{3}$ interference is multiplied by a factor of 100 for better visibility, i.e. this contribution is strongly suppressed compared to the other contributions and can safely be neglected. This suppression is caused by two effects: by the relatively large mass difference $\Delta m_{23}=m_{3}-m_{2}$ and by the choice of parameters in scenario 3, which leads to cancellations due to opposite-sign Yukawa couplings. (For details see [8].)

These observations serve as a guidance for the calculation of the NLO corrections, which are outlined in the next section.

\section{Next-to-leading order corrections}

Because $p p \rightarrow \phi \rightarrow t \bar{t}$ is already a one-loop process at LO the NLO corrections involve a twoloop calculation. We have chosen a simplified approach and computed the NLO corrections to $\phi_{j}$ $(j=2,3)$ production in the heavy top mass limit which reduces the top-quark loop-induced $g g \phi$ coupling to a local vertex. This limit can be consistently described in the context of an effective field theory that yields local effective Higgs gluon couplings:

$$
\mathcal{L}_{\mathrm{eff}}=\sum_{j=2,3}\left[f_{S j} G_{\mu \nu}^{a} G_{a}^{\mu \nu}+f_{P j} \epsilon_{\mu \nu \alpha \beta} G_{a}^{\mu \nu} G_{a}^{\alpha \beta}\right] \phi_{j}
$$

where $f_{S j}$ and $f_{P j}$ are the Wilson coefficients describing the coupling of the scalar and pseudoscalar component of the Higgs boson $\phi_{j}$ to gluons. The effective Lagrangian in Eq. (5.1) also contains couplings of $\phi_{j}$ to three and four gluons, of which only the former is relevant for heavy Higgs production and decay to $t \bar{t}$ at NLO. In the heavy top-mass limit the two-loop calculation becomes effectively a one-loop calculation. This limit can also be understood as the first order in an expansion in $m_{\phi} / m_{t}$. In the case of Higgs production it has been shown [14] that even for $m_{\phi}>m_{t}$ this approach gives results with rather small uncertainties of about $10 \%$ depending on the size of $m_{\phi}$ if an appropriate $K$ factor is applied to the NLO corrections in the effective theory. We apply this procedure in the following way in order to get an approximate result for the cross section of our process at NLO QCD:

$$
\sigma_{\mathrm{NLO}}^{\mathrm{approx}} \equiv \sum_{j=2,3}\left(\sigma_{\text {full }, j}^{(0)}+\sigma_{\text {full }, j, \mathrm{QCD}}^{(0)}+K_{j} \sigma_{\mathrm{eff}, j}^{(1)}+\sigma_{\mathrm{eff}, j, \mathrm{QCD}}^{(1)}\right) \quad \text { with } \quad K_{j}=\frac{\sigma_{\text {full, } j}^{(0)}}{\sigma_{\mathrm{eff}, j}^{(0)}}
$$


Here $\sigma_{\text {full }, j}^{(0)}$ denote the LO cross sections for

$$
p p \rightarrow \phi_{j} \rightarrow t \bar{t}
$$

and $\sigma_{\text {full,QCD }}^{(0)}$ results from the interference of the amplitude of (5.3) and of the QCD background at LO. The subscript full indicates that the full top-quark mass dependence is kept. The terms $\sigma_{\mathrm{eff}, j}^{(1)}$ $\left(\sigma_{\mathrm{eff}, j}^{(0)}\right)$ and $\sigma_{\mathrm{eff}, j, \mathrm{QCD}}^{(1)}$ represent the NLO (LO) cross sections for the process (5.3) and the interference with the QCD background at NLO, respectively, in the effective theory. While Eq. (5.2) is given for inclusive cross sections an analogous formula can also be applied to individual bins of differential distributions.

In addition to the heavy top limit we apply a second approximation. As the LO results in Sec. 4 show, the dominant contribution from heavy Higgs bosons to $t \bar{t}$ production comes from the resonant region. Hence we restrict the NLO calculation of the differential distributions to this region. The calculation involves factorizable and non-factorizable QCD corrections to LO Higgs production and decay. The former are manifestly resonant while the latter consist of resonant and non-resonant contributions. The resonant parts of the non-factorizable contributions can be extracted by applying the soft gluon approximation $[15,16,17,18]$. Within this approximation non-factorizable contributions from the real and virtual corrections cancel exactly. Thus one is left with only factorizable contributions. (For details see [8].)

Apart from the two approximations we apply standard techniques to perform the NLO calculation. In particular, we use the dipole subtraction method $[19,20]$ to handle the infrared divergences in the real and virtual corrections. Analytical results of our NLO calculation of the squared matrix elements are given in [8].

\section{Results}

In this section we present the results for heavy Higgs production and decay to $t \bar{t}$ including the approximate NLO QCD corrections to the 2HDM and 2HDM-QCD interference contributions, as well as the full NLO QCD and weak interaction corrections to the background.

The inclusive cross sections for $t \bar{t}$ production are listed in Tab. 2 for the three parameter scenarios. The label $2 \mathrm{HDM}$ denotes the contribution to the inclusive $t \bar{t}$ cross section from the two heavy Higgs bosons and the interference with the QCD background at NLO using the aforementioned approximations, while $\sigma_{\mathrm{QCDW}}$ is the contribution of the QCD background at NLO including the weak interaction corrections. The ratio $\sigma_{2 \mathrm{HDM}} / \sigma_{\mathrm{QCDW}}$ is rather small; the $2 \mathrm{HDM}$ contribution is about $2 \%$ of the inclusive QCD cross section in the case of scenario 1 and about $1 \%$ for scenarios 2 and 3. There are mainly two effects causing the inclusive cross section to be hardly sensitive to the heavy Higgs resonances. The QCD-induced $t \bar{t}$ production is large and the interference effects partly cancel the resonant enhancement of the Higgs contributions. It is therefore important to study other, more sensitive observables, in particular observables evaluated in $t \bar{t}$ invariant mass bins.

The $t \bar{t}$ invariant mass distribution $d \sigma / d M_{t \bar{t}}$ is shown in Fig. 2 for scenarios 1-3. The upper plots display the SM $M_{t \bar{t}}$ distribution and the sum of SM and 2HDM contribution ("squared" and interference) at NLO including the uncertainty due to renormalization and factorization scale variations. 


\begin{tabular}{l|lll} 
& Scenario 1 & Scenario 2 & Scenario 3 \\
\hline$\mu_{0}[\mathrm{GeV}]$ & 265 & 312.5 & 325 \\
$\sigma_{\mathrm{QCDW}}[\mathrm{pb}]$ & $643.22_{-77.71}^{+81.23}$ & $624.25_{-76.19}^{+80.98}$ & $619.56_{-75.72}^{+81.05}$ \\
$\sigma_{2 \mathrm{HDM}}[\mathrm{pb}]$ & $13.59_{-1.64}^{+1.85}$ & $7.4_{-0.78}^{+0.77}$ & $7.21_{-0.77}^{+0.81}$ \\
\hline$\sigma_{2 \mathrm{HDM}} / \sigma_{\mathrm{QCDW}}[\%]$ & 2.1 & 1.2 & 1.2
\end{tabular}

Table 2: Inclusive $t \bar{t}$ cross sections without and with the heavy Higgs resonances.

The lower plots in Fig. 2 show the ratio (QCDW + 2HDM)/QCDW at NLO (red) and at LO (green). The strongest effect of about $7 \%$ appears in those bins of the $M_{t \bar{t}}$ distribution in scenario 1 where the contributions of the two resonances overlap. In Fig. 3 we show a comparison of our result for
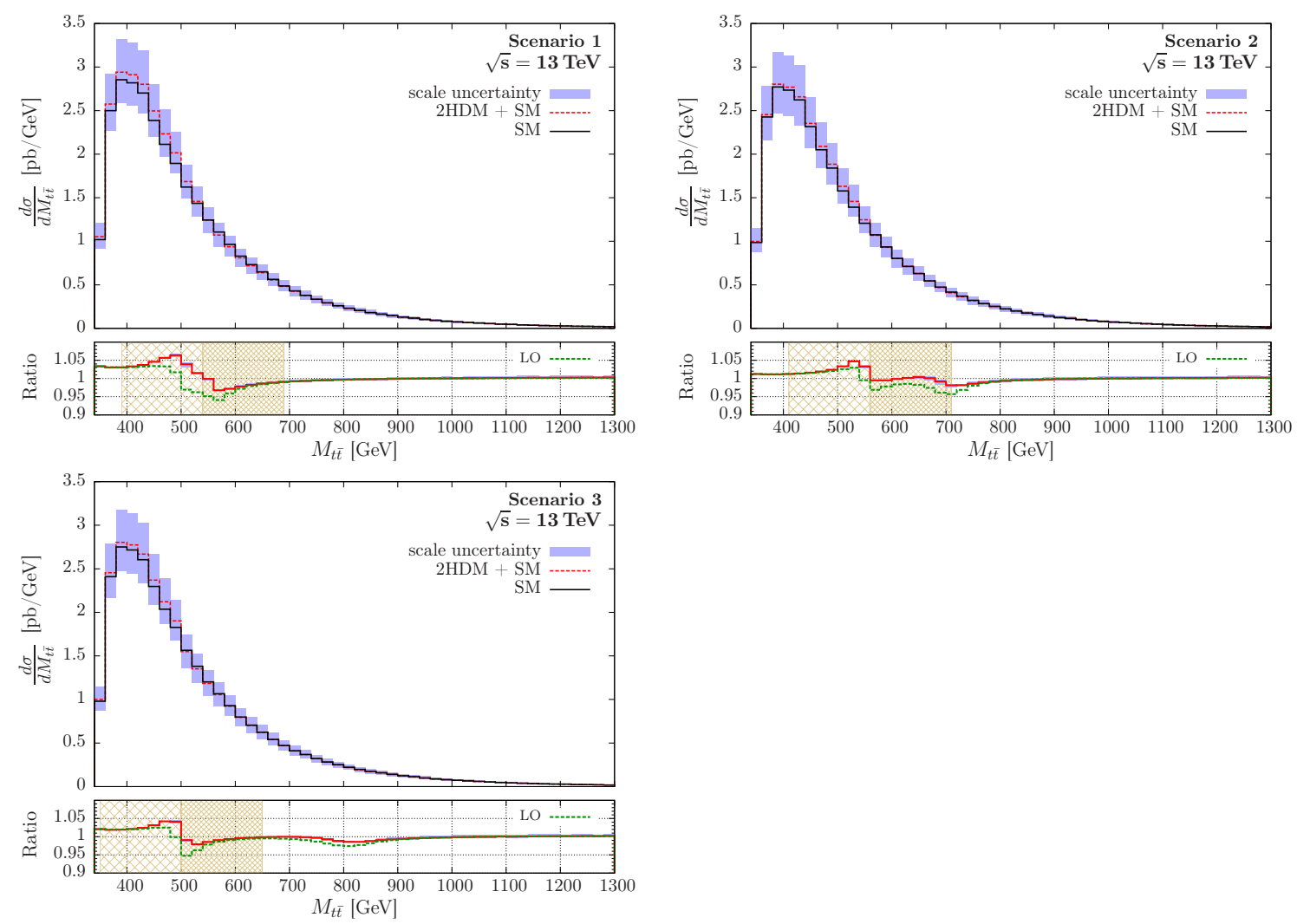

Figure 2: $M_{t \bar{t}}$ distribution for scenario 1-3. Upper left: scenario 1, upper right: scenario 2, lower plot: scenario 3 .

the $M_{t \bar{t}}$ distribution within scenario 1 with a CMS analysis [6] at $8 \mathrm{TeV}$ center-of-mass energy using $19.7 \mathrm{fb}^{-1}$ of data. As can be seen from Fig. 3 this analysis is not yet sensitive enough to constrain scenario 1. A similar analysis from ATLAS exists ${ }^{1}$ [5] with a similar sensitivity. In Fig. 3 one can see that the background uncertainty of the analysis [6] is too large to detect the signal. Furthermore the large bin size of $100 \mathrm{GeV}$ causes the theoretical signal-to-background ratio to fall from a peak

\footnotetext{
${ }^{1}$ The ATLAS analysis [5] of the $M_{t \bar{t}}$ distribution at $8 \mathrm{TeV}$ is also not sensitive enough to constrain this model because the interference effects were not taken into account. However, during the writeup of this proceedings contribution an updated ATLAS analysis [21] was published. This analysis is considerably more sensitive for several reasons. It succeeds in reducing the uncertainty on the background, it uses a smaller $M_{t \bar{t}}$ bin width and takes interference effects into account.
} 
value of about $7 \%$ to below $5 \%$.

By imposing cuts on $M_{t \bar{t}}$ (indicated by hatched regions in Fig. 2) below and above the resonance

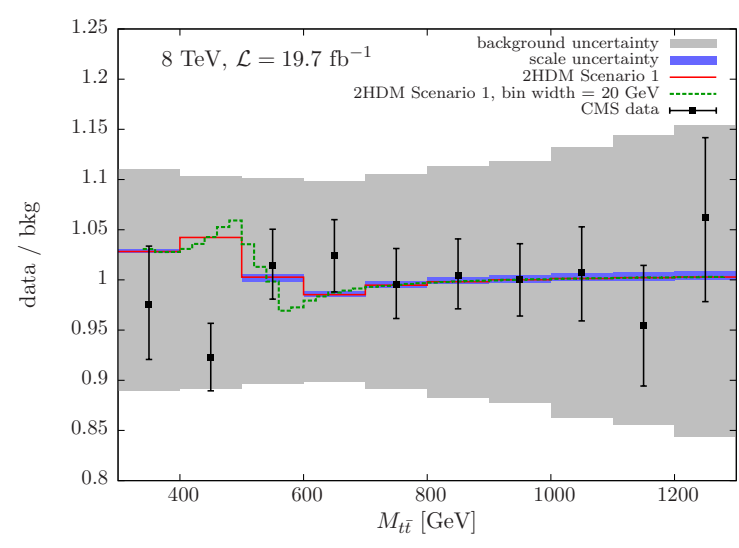

Figure 3: CMS result for the ratio data/bkg. binned in $M_{t \bar{t}}$ at $\sqrt{s}=8 \mathrm{TeV}$ taken from [6]. The theoretical prediction for scenario 1 is plotted in red (100 GeV binning) and green (20 GeV binning).

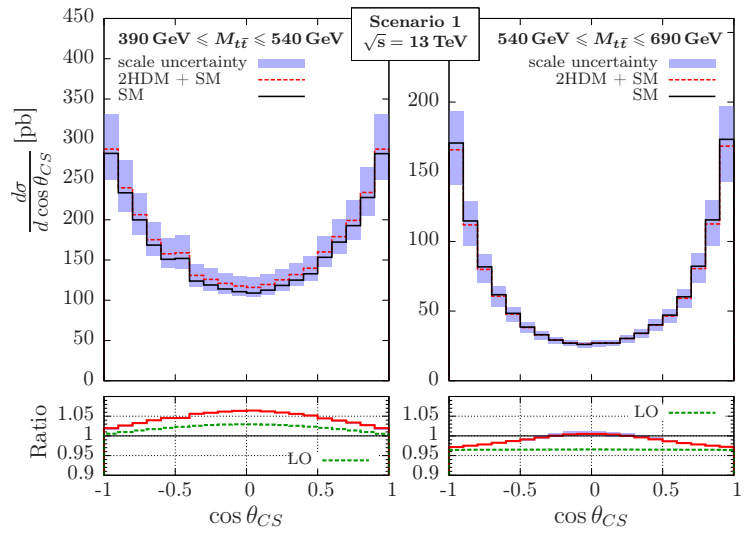

Figure 4: Distribution of the Collins-Soper angle for scenario 1 with two different $M_{t \bar{t}}$ cuts.

we avoid the peak-dip cancellation and obtain an estimate on the largest possible Higgs effects in different observables. Besides the $M_{t \bar{t}}$ distribution we studied the distribution of the top-quark rapidity, of the top-quark transverse momentum, and of the Collins-Soper angle $\theta_{\mathrm{CS}}$. Among these observables the distribution $d \sigma / d \cos \theta_{\mathrm{CS}}$ is the most sensitive one to a heavy Higgs boson. This distribution is shown in Fig. 4 for scenario 1 for two different $M_{t \bar{t}}$ cuts. The lower plots show the signal-to-background ratio which is $>5 \%$ between $\cos \theta_{\mathrm{CS}}=-0.4$ and $\cos \theta_{\mathrm{CS}}=0.5$ in the lower $M_{t \bar{t}}$ bin. Furthermore, Fig. 4 illustrates the importance of the NLO corrections which lead to an enhancement of the signal-to-background ratio.

\section{Conclusion}

We calculated the NLO QCD corrections to the resonant production of heavy Higgs bosons at the LHC and their decay into top-quark pairs in the large top mass limit. We have taken into account the SM $t \bar{t}$ continuum and its interference with the signal at NLO. This interference has an important effect on the shape of the $t \bar{t}$ invariant mass distribution, $M_{t \bar{t}}$, in the vicinity of a heavy Higgs resonance. Depending on the mass and the couplings of the resonance it leads to a peak-dip structure or to a dip rather than a bump in the $M_{t \bar{t}}$ spectrum. We found that the QCD corrections to these $M_{t \bar{t}}$ shape distortions, but also to the distribution of the Collins-Soper angle, are significant in the resonance region. We investigated these heavy Higgs-boson effects within a type-II two-Higgsdoublet extension of the SM.

The shape-distortions of the $M_{t \bar{t}}$ spectrum predicted at NLO QCD have important consequences for the search for a heavy Higgs-boson signal in the measured $t \bar{t}$ invariant mass distribution. If this distribution is measured with a large bin size the peak and dip structure caused by a Higgs resonance averages out. As a consequence the inclusive $t \bar{t}$ cross section is affected by the heavy 
Higgs-boson contributions in our scenarios only at the level of 1-2\%. A dedicated resonance search could be done by scanning the measured $t \bar{t}$ invariant mass spectrum with a sliding $M_{t \bar{t}}$ window with the smallest experimentally attainable width.

\section{References}

[1] ATLAS collaboration, G. Aad et al., Observation of a new particle in the search for the Standard Model Higgs boson with the ATLAS detector at the LHC, Phys. Lett. B716 (2012) 1-29, [1207.7214].

[2] CMS collaboration, S. Chatrchyan et al., Observation of a new boson at a mass of $125 \mathrm{GeV}$ with the CMS experiment at the LHC, Phys. Lett. B716 (2012) 30-61, [1207. 7235].

[3] CMS collaboration, V. Khachatryan et al., Precise determination of the mass of the Higgs boson and tests of compatibility of its couplings with the standard model predictions using proton collisions at 7 and 8 TeV, Eur. Phys. J. C75 (2015) 212, [1412.8662].

[4] ATLAS collaboration, G. Aad et al., Measurements of the Higgs boson production and decay rates and coupling strengths using pp collision data at $\sqrt{s}=7$ and $8 \mathrm{TeV}$ in the ATLAS experiment, Eur. Phys. J. C76 (2016) 6, [1507.04548].

[5] ATLAS collaboration, G. Aad et al., A search for tt resonances using lepton-plus-jets events in proton-proton collisions at $\sqrt{s}=8 \mathrm{TeV}$ with the ATLAS detector, JHEP 08 (2015) 148, [1505.07018].

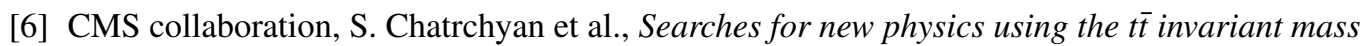
distribution in pp collisions at $\sqrt{s}=8 \mathrm{TeV}$, Phys. Rev. Lett. 111 (2013) 211804, [1309.2030].

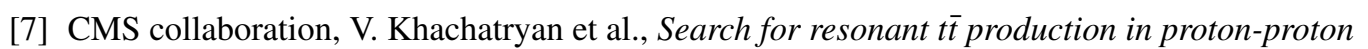
collisions at $\sqrt{s}=8 \mathrm{TeV}$, Phys. Rev. D93 (2016) 012001, [1506.03062].

[8] W. Bernreuther, P. Galler, C. Mellein, Z. G. Si and P. Uwer, Production of heavy Higgs bosons and decay into top quarks at the LHC, Phys. Rev. D93 (2016) 034032, [1511. 05584].

[9] B. Hespel, F. Maltoni and E. Vryonidou, Signal background interference effects in heavy scalar production and decay to a top-anti-top pair, 1606.04149.

[10] G. C. Branco, P. M. Ferreira, L. Lavoura, M. N. Rebelo, M. Sher and J. P. Silva, Theory and phenomenology of two-Higgs-doublet models, Phys. Rept. 516 (2012) 1-102, [1106.0034].

[11] F. Mahmoudi and O. Stal, Flavor constraints on the two-Higgs-doublet model with general Yukawa couplings, Phys. Rev. D81 (2010) 035016, [0907.1791].

[12] T. Hermann, M. Misiak and M. Steinhauser, $\bar{B} \rightarrow X_{s} \gamma$ in the Two Higgs Doublet Model up to Next-to-Next-to-Leading Order in QCD, JHEP 11 (2012) 036, [1208.2788].

[13] O. Eberhardt, U. Nierste and M. Wiebusch, Status of the two-Higgs-doublet model of type II, JHEP 07 (2013) 118, [1305 . 1649].

[14] M. Kramer, E. Laenen and M. Spira, Soft gluon radiation in Higgs boson production at the LHC, Nucl. Phys. B511 (1998) 523-549, [hep-ph/9611272].

[15] V. S. Fadin, V. A. Khoze and A. D. Martin, Interference radiative phenomena in the production of heavy unstable particles, Phys. Rev. D49 (1994) 2247-2256.

[16] K. Melnikov and O. I. Yakovlev, Final state interaction in the production of heavy unstable particles, Nucl. Phys. B471 (1996) 90-120, [hep-ph/9501358].

[17] W. Beenakker, A. P. Chapovsky and F. A. Berends, Nonfactorizable corrections to W pair production: Methods and analytic results, Nucl. Phys. B508 (1997) 17-63, [hep-ph/9707326].

[18] S. Dittmaier, A. Huss and C. Schwinn, Mixed QCD-electroweak $O\left(\alpha_{s} \alpha\right)$ corrections to Drell-Yan processes in the resonance region: pole approximation and non-factorizable corrections, Nucl. Phys. B885 (2014) 318-372, [1403.3216].

[19] S. Catani and M. H. Seymour, A General algorithm for calculating jet cross-sections in NLO QCD, Nucl. Phys. B485 (1997) 291-419, [hep-ph/9605323].

[20] S. Catani, S. Dittmaier, M. H. Seymour and Z. Trocsanyi, The Dipole formalism for next-to-leading order QCD calculations with massive partons, Nucl. Phys. B627 (2002) 189-265, [hep-ph/0201036].

[21] ATLAS collaboration, Search for heavy Higgs bosons A/H decaying to a top-quark pair in pp collisions at $\sqrt{s}=8 \mathrm{TeV}$ with the ATLAS detector, ATLAS-CONF-2016-073. 